\title{
Taquicardia supraventricular como manifestación inicial de polimiositis. Reporte de caso
}

\author{
Supraventricular tachycardia as initial manifestation of polymyositis. A case report
}

\author{
Jorge E. Reyes-Tovilla1,2,*, Fabio Solis-Jiménez ${ }^{1,2}$ y César L. González-Aguilar ${ }^{1,3}$ \\ ${ }^{1}$ Servicio de Medicina Interna, Hospital General de México Dr. Eduardo Liceaga, Facultad de Medicina, Universidad Nacional Autónoma de México; \\ ${ }^{2}$ Instituto Nacional de Cardiología "Ignacio Chávez; ${ }^{3}$ Centro Médico Nacional "20 de Noviembre", Universidad Nacional Autónoma de México, Ciudad \\ de México, México
}

Las miopatías inflamatorias son un grupo de enfermedades autoinmunes del músculo esquelético que pueden afectar otros órganos y sistemas, como el aparato cardiovascular ${ }^{1}$, siendo el más afectado el miocardio, por ateroesclerosis coronaria. Las anormalidades del ritmo como la taquicardia supraventricular son raras y se presentan en el $0.9 \%$ de los casos, las más frecuentes son los trastornos del sistema de conducción como los bloqueos de rama y anormalidades de la repolarización ${ }^{2}$. Presentamos el caso de un hombre de 41 años sin antecedentes de importancia. Ingresó por dolor precordial, de inicio súbito, de una hora de duración. Se documentó taquicardia de QRS estrecho con RP largo (Fig. 1), que revirtió con adenosina y se documentó posteriormente ritmo sinusal con bloqueo de rama derecha (Fig. 2). En el Servicio de Medicina Interna se inició protocolo por miopatía inflamatoria, en la exploración física con debilidad proximal de músculos del cuello y las extremidades, además de elevación de enzimas musculares (CK 2,090 U/I). Se solicitaron autoanticuerpos: anticuerpos antinucleares 1:160 patrón citoplásmico, anti-Jo-1 $0.52 \mathrm{U} / \mathrm{ml}$, anti-Ro52 $150 \mathrm{U} / \mathrm{ml}$, troponina I $0.47 \mathrm{pg} / \mathrm{ml}$, isoenzima MB $89 \mathrm{U} / \mathrm{l}$ y péptido natriurético de tipo B 1,261 pg/ml. Se realizó ecocardiograma transtorácico, con fracción de expulsión del ventrículo izquierdo del $65 \%$, presión sistólica del ventrículo derecho 35, TAPSE (excursión sistólica del anillo tricúspide) 15, onda $S$ tricuspídea $9 \mathrm{~cm} / \mathrm{s}$. Biopsia de músculo en la que se observaron fibras musculares atróficas y miocitos con el núcleo centralizado. Velocidades de neuroconducción con descargas complejas repetitivas, potenciales de fibrilación y ondas positivas. Se realizó espirometría (Fig. 3) y tomografía de tórax (Fig. 4), por sospecha de neumopatía intersticial. Cumplió criterios de Bohan y Peter para polimiositis y se inició metotrexato, prednisona, metoprolol y propafenona. Las anormalidades del ritmo son una presentación atípica de la polimiositis y se han realizado pocos estudios para conocer su prevalen$\mathrm{cia}^{2,3}$. No se han encontrado alteraciones morfológicas en el ecocardiograma al momento de la presentación en un estudio con 30 pacientes $^{4}$, sin embargo en nuestro caso solo la TAPSE es la alterada. El estado proinflamatorio de la enfermedad autoinmune podría asociarse a las anormalidades del ritmo, pero aún faltan muchos estudios que comprueben esta situación, la taquicardia supraventricular es una manifestación atípica en la polimiositis.

\footnotetext{
Correspondencia:

*Jorge E. Reyes-Tovilla

E-mail: drjert88@gmail.com

Fecha de recepción: 10-02-2020

Fecha de aceptación: 08-05-2020 DOI: 10.24875/ACM.20000054

Disponible en internet: 05-08-2020 Arch Cardiol Mex. 2021;91(2):235-237 www.archivoscardiologia.com CC BY-NC-ND (http://creativecommons.org/licenses/by-nc-nd/4.0/).
} 


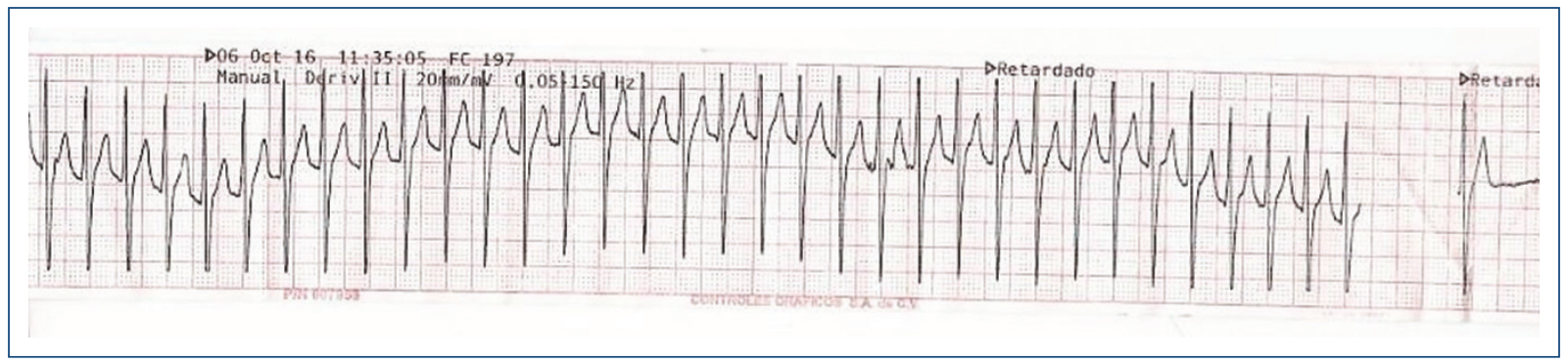

Figura 1. Tira de electrocardiograma DII al ingreso.

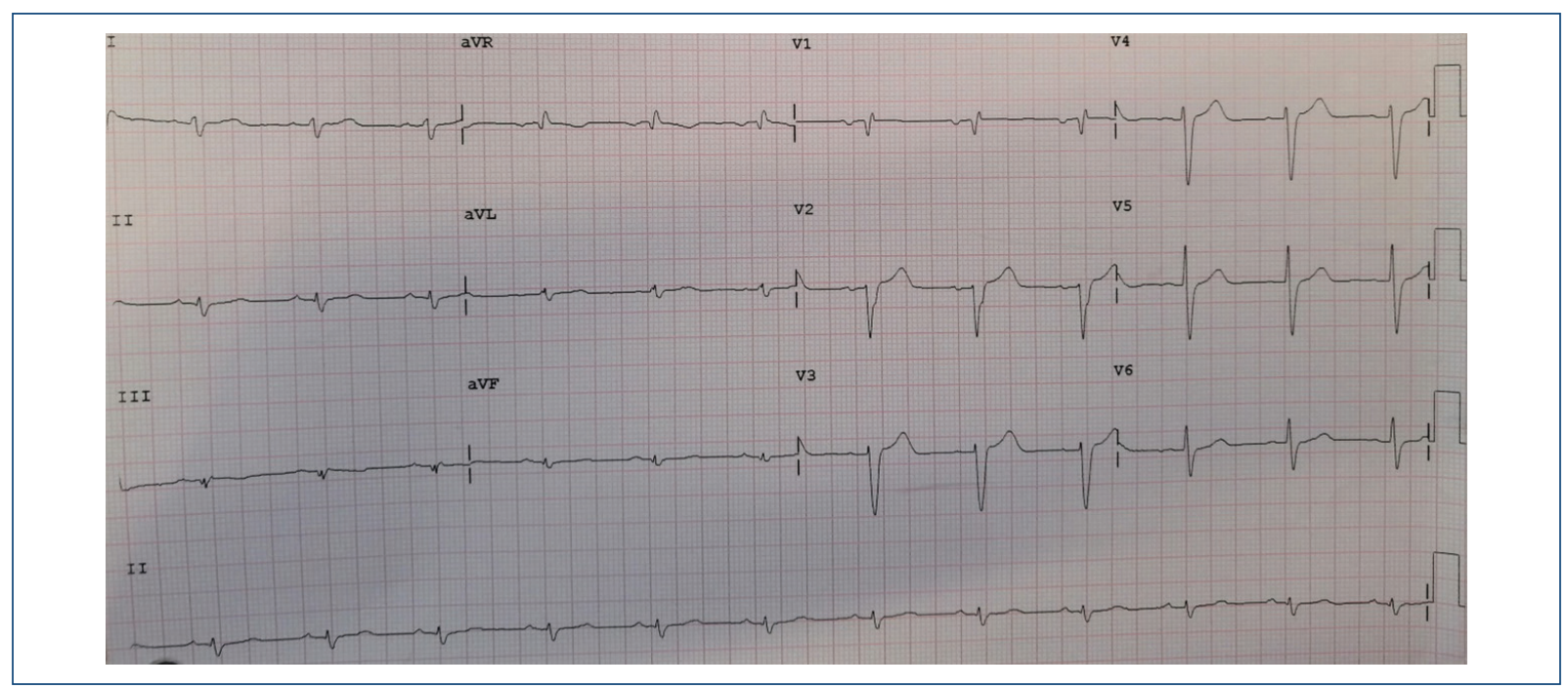

Figura 2. Electrocardiograma de 12 derivaciones posterior a la adenosina.

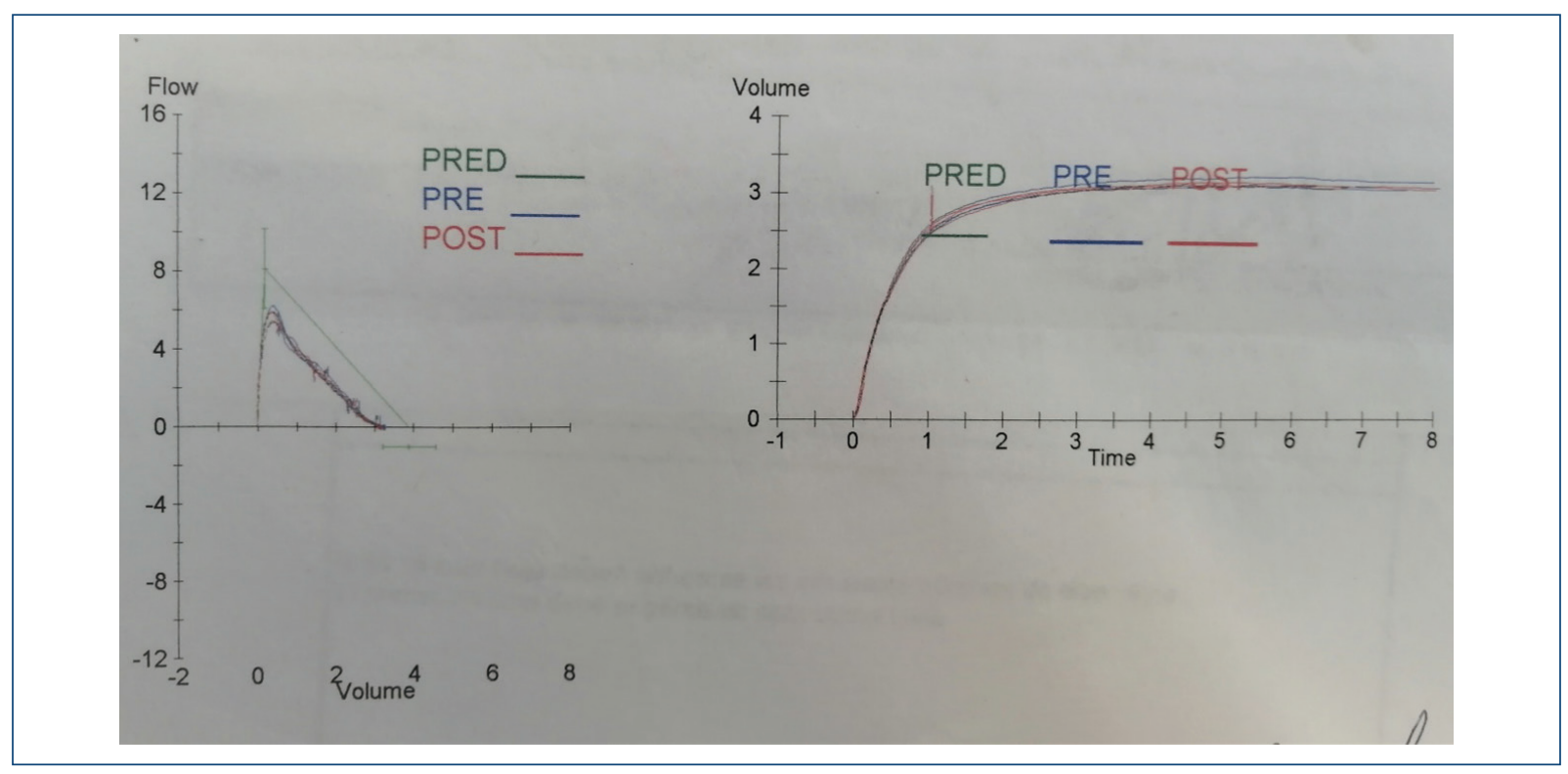

Figura 3. Espirometría que sugiere restricción moderada con respuesta al broncodilatador. 


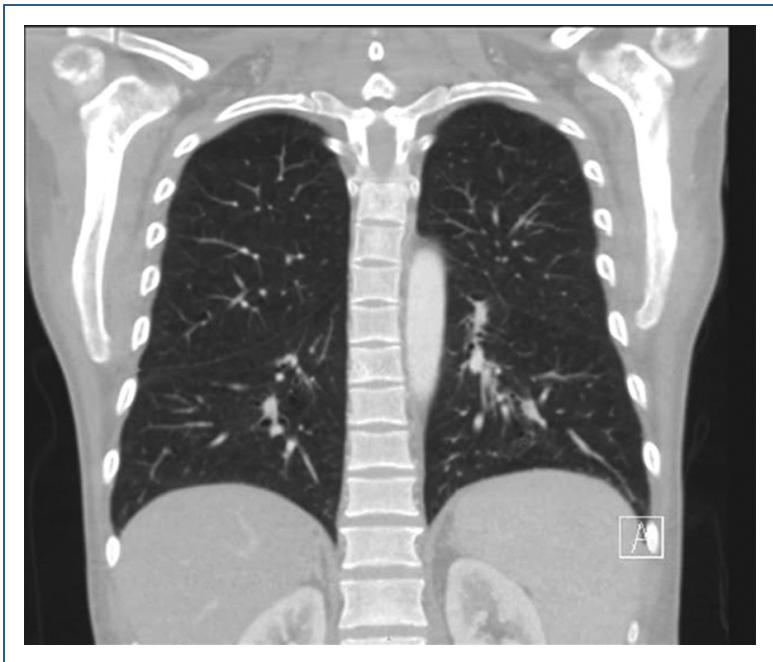

Figura 4. Tomografía de alta resolución. Corte axial, ventana pulmonar, que sugiere enfermedad pulmonar intersticial.

\section{Agradecimientos}

A nuestras familias, a Juan D. Muñoz, Alejandra Aquino y a la institución por el apoyo en estos cuatro años de residencia de medicina interna.

\section{Financiamiento}

Financiamiento por Hospital General de México Dr. Eduardo Liceaga.

\section{Conflicto de intereses}

Los autores declaran no tener conflicto de intereses.

\section{Responsabilidades éticas}

Protección de personas y animales. Los autores declaran que para esta investigación no se han realizado experimentos en seres humanos ni en animales.

Confidencialidad de los datos. Los autores declaran que han seguido los protocolos de su centro de trabajo sobre la publicación de datos de pacientes.

Derecho a la privacidad y consentimiento informado. Los autores declaran que en este artículo no aparecen datos de pacientes.

\section{Bibliografía}

1. Danieli MG, Gelardi C, Guerra F, Cardinaletti P, Pidini V, Gabrielli A. Cardiac involvement in polymyositis and dermatomyositis. Autoimmun Rev. 2016;15(5):462-5.

2. Deveza LM, Miossi R, de Souza FH, Shimabuco AY, Favarato MH, Grindler J, et al. Electrocardiographic changes in dermatomyositis and polymyositis. Rev Bras Reumatol Engl Ed. 2016;56(2):95-100.

3. Stern R, Godbold JH, Chess Q, Kagen LJ. ECG abnormalities in polymyositis. Arch Intern Med. 1984;144(11):2185-9.

4. Péter A, Balogh A, Szilágyi S, Faludi R, Nagy-Vincze M, Édes I, et al. Echocardiographic abnormalities in new-onset polymyositis/dermatomyositis. J Rheumatol. 2015;42(2):272-81. 